\title{
Canoparmelia y Crespoa (Parmeliaceae, Ascomycota) en EL NORDESTe de Argentina; Canoparmelia Caroliniana y $C$. CRYPTOCHLOROPHAEA NUEVAS CITAS PARA ARGENTINA
}

\begin{abstract}
ANDREA MICHLIG ${ }^{1}$
Summary: Canoparmelia and Crespoa (Parmeliaceae, Ascomycota) in northeastern Argentina; Canoparmelia caroliniana and C. cryptochlorophaea new records to Argentina. A study of the genera Canoparmelia and Crespoa in northeastern Argentina is presented. Seven species were studied, including Canoparmelia caroliniana and C. cryptochlorophaea as the first reports for Argentina, and C. austroamericana for the region. The known geographic distribution of $C$. texana and Crespoa crozalsiana is also extended. Descriptions, illustrations, and a key for the identification to differentiate the treated species are included.
\end{abstract}

Key words: Lichens, biodiversity, protected areas.

Resumen: Se presenta un estudio de los géneros Canoparmelia y Crespoa en el Nordeste de Argentina. Siete especies fueron estudiadas, incluyendo Canoparmelia caroliniana y C. cryptochlorophaea como primeros registros para Argentina y $C$. austroamericana para la región. Se extiende además el área de distribución geográfica conocida de C. texana y Crespoa crozalsiana. Se incluyen descripciones detalladas, ilustraciones y una clave para diferenciar las especies tratadas.

Palabras clave: Líquenes, biodiversidad, áreas protegidas.

\section{INTRODUCCIÓN}

Canoparmelia Elix \& Hale fue originalmente descripto como un género derivado de Pseudoparmelia Lynge, para agrupar especies con los lóbulos relativamente angostos [(0,5-) 3,0-5,0 $(-8,0) \mathrm{mm}$ de ancho], subirregulares a sublineares, con ápice subredondeado a redondeado y margen eciliado, una superficie superior gris mineral cubierta por una epicorteza con poros, paredes celulares con isoliquenano, ricinas simples, apotecios adnatos a subestipitados con disco imperforado y conidios bifusiformes a raramente cilíndricos, baciliformes, fusiformes o filiformes (Hale, 1974, 1976; Elix et al., 1986; Elix, 1993, 1994). Con estos caracteres se establecía la delimitación de Canoparmelia sensu lato que actualmente ha quedado bastante restringida.

Los estudios filogenéticos recientes en Parmeliaceae basados en datos moleculares indicaron

\footnotetext{
1 Instituto de Botánica del Nordeste (IBONE-CONICET), Sargento Cabral 2131, C.C. 209, C.P. 3400, Corrientes, Argentina. e-mail: andrea.michlig@yahoo.com
}

que Canoparmelia, de acuerdo a su circunscripción tradicional, es un género polifilético (Crespo et al., 2010a). Esto dio como resultado la creación de dos nuevos géneros: Austroparmelina Crespo, Divakar \& Elix, a donde fueron transferidas varias especies anteriormente ubicadas en Canoparmelia (C. macrospora, C. norpruinata, C. pruinata, $C$. subarida y C. subtiliacea) y las especies australásicas de Parmelina Hale (Crespo et al., 2010b), y Crespoa (D. Hawksw.) Lendemer \& Hodkinson, constituido por cuatro especies pertenecientes al grupo de Canoparmelia crozalsiana (C. carneopruinata, C. crozalsiana, $C$. inhaminensis y $C$. schelpei), que constituye un linaje hermano a Parmotrema (Crespo et al., 2010a; Hawksworth, 2011; Lendemer \& Hodkinson, 2012). A pesar de que Crespoa ha sido separado de Canoparmelia al nivel de género, no se han señalado aún caracteres morfológicos ni anatómicos claramente distintivos que permitan diferenciarlos más allá de los estudios moleculares y requieren estudios adicionales (Crespo et al., 2010a, 2011).

De acuerdo al último catálogo de líquenes 
de Argentina (Calvelo \& Liberatore, 2002), se encuentran ya citadas cinco especies de estos géneros en el país, tres de Canoparmelia y dos de Crespoa, todas ellas representadas en el NEA. El objetivo de este trabajo es contribuir al conocimiento de ambos géneros en el Nordeste de Argentina. Se han estudiado siete especies de las cuales dos se mencionan por primera vez para Argentina y una para la región, y se extiende el área de distribución geográfica de otras dos especies. Se presentan descripciones de todas ellas, comentarios sobre sus caracteres diagnósticos y diferencias con especies relacionadas, área de distribución geográfica e ilustraciones. También se presenta una clave para diferenciar dichas especies.

\section{Materiales y Métodos}

Se han estudiado 70 especímenes, depositados en el herbario CTES (Thiers, 2013), que fueron coleccionados recientemente por la autora en distintas localidades de las provincias de Chaco, Corrientes, Formosa y Misiones. Debido al alto número de especímenes estudiados, sólo se menciona uno por localidad, a menos que haya información diferente en relación al ambiente o sustrato.
La morfología del talo fue estudiada bajo microscopio estereoscópico (Leica MZ6). Para la observación de las ascosporas y conidios se realizaron cortes finos a mano alzada de los apotecios y picnidios, posteriormente montados en hidróxido de potasio al $5 \%$ y floxina acuosa al $1 \%$ para su estudio en microscopio óptico (Leica CME). Las mediciones fueron realizadas con aumentos de 400x y 1000x.

Las sustancias liquénicas se identificaron de manera preliminar mediante reacciones puntuales de color con hidróxido de potasio al 10\% (K), hipoclorito de sodio (C) y K seguido de $\mathrm{C}(\mathrm{KC})$, fluorescencia en cámara de luz ultravioleta y finalmente cada compuesto fue caracterizado de manera más precisa mediante cromatografía en capa delgada (CCD) con solventes A y C, por comparación con muestras cuyas sustancias eran conocidas (White \& James, 1985; Orange et al., 2010). Esta caracterización química se describe para cada especie bajo el ítem Sustancias químicas.

A lo largo de este trabajo, se mencionan los nombres genéricos para cada especie en forma abreviada, utilizando $C$. para Canoparmelia y $C r$. para Crespoa. Los autores de los nombres científicos se escriben de acuerdo a The International Plant Names Index (2012).

\section{Resultados y Discusión}

\section{Clave de las especies de Canoparmelia y Crespoa del Nordeste de Argentina}

1. Propágulos vegetativos ausentes ................................................................................................ 2

2. Médula $\mathrm{K}+$ amarillo, $\mathrm{KC}$ - (con ácido estíctico); apotecios con disco pruinoso; superficie inferior negra; conidios filiformes

C. scrobicularis

2'. Médula K-, KC+ rosa pálido (con ácido perlatólico); apotecios con disco epruinoso; superficie inferior castaña oscura a clara; conidios fusiformes a bifusiformes

C. austroamericana

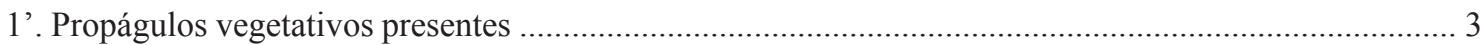

3. Talo con isidios; médula $\mathrm{KC}+$ rosa (con ácido perlatólico)

C. caroliniana

3'. Talo con soredios; médula KC- o + rosa (química diferente) ........................................................ 4

4. Soralios marginales a submarginales, notoriamente capitados; médula $\mathrm{K}+$ rosa pálido (con ácido criptoclorofaeico)

C. cryptochlorophaea

4'. Soralios laminares, orbiculares; médula K- o + (química diferente)

5

5. Superficie superior lisa a suavemente crestada; médula $\mathrm{K}-, \mathrm{KC}+$ rosa (con ácido divaricático) ... C. texana

5'. Superficie superior fuertemente foveolada; médula K+ amarillo, KC- (con ácido estíctico) .... 6 6. Lóbulos 2-4 mm de ancho Cr. crozalsiana

6'. Lóbulos 0,5-2 mm de ancho

Cr. carneopruinata 
1. Canoparmelia austroamericana Adler, Mycotaxon 28: 251. 1987. (Fig. 1, A-E)

Talo folioso, gris mineral, coriáceo, corticícola, moderada a fuertemente sujeto al sustrato, 4-5 mm diám.; lóbulos subirregulares, de ramificación irregular, 2-3 $\mathrm{mm}$ de ancho, levemente imbricados, de ápice redondeado; margen entero, eciliado. Superficie superior lustrosa, fuertemente foveolada, tornándose verrucosa hacia el centro del talo, continua, maculada; máculas irregulares a reticulares sobre las crestas, laminares, más conspicuas hacia el ápice de los lóbulos. Isidios, soralios, pústulas y dáctilos ausentes. Médula blanca. Superficie inferior castaña oscura a clara, mate, lisa a levemente rugosa, moderadamente ricinada, con una angosta zona marginal castaña, desnuda, lisa a rugosa; ricinas simples, castañas claras a blanquecinas, distribuidas irregularmente. Apotecios abundantes, cupuliformes a urceolados, 1,5-5 mm diám., laminares, margen entero a crenado, anfitecio foveolado, disco castaño oscuro, epruinoso, imperforado; ascosporas elipsoidales, 8-12 x 4-7 $\mu \mathrm{m}$. Picnidios abundantes, submarginales a laminares, de ostíolo negro; conidios bifusiformes, a veces levemente, a fusiformes, 5-8 $\mu \mathrm{m}$ de largo.

Sustancias químicas. Corteza K+ amarillo, UV[con atranorina (menor)], médula $\mathrm{K}-, \mathrm{C}-, \mathrm{KC}+$ rosa pálido, UV+ blanco azulado [con ácidos perlatólico (mayor), estenospórico (menor) y glomeriférico (menor)].

Material examinado. ARGENTINA. Prov. Chaco: Dpto. Libertador General San Martín, Ruta provincial $\mathrm{N}^{\mathrm{o}} 3$, entre Presidencia Roca y la intersección de la Ruta Prov. $\mathrm{N}^{\circ}$ 90; camino a La Escuela EGB No $564,26^{\circ} 13$ ' 5' $\mathrm{S} 59^{\circ} 29^{\prime} 42,5^{\prime}$ 'W, $75 \mathrm{msnm}$, sobre postes de alambrado, 12/II/2010, Michlig \& Niveiro 2309 (CTES).

Distribución geográfica. Fue registrada únicamente en Argentina, donde fue citada para las provincias de Buenos Aires (Adler, 1987) y Santiago del Estero (Adler, 1987, 2013), y Chile (Quilhot et al., 1998). Se registra ahora por primera vez para el NEA.

Observaciones. Canoparmelia austroamericana es una de las dos especies de Canoparmelia de Argentina que carecen de propágulos vegetativos. Se caracteriza además por la superficie superior fuertemente foveolada (Fig. 1 A-B, D), tornándose verrucosa hacia el centro del talo, con máculas irregulares a reticulares más conspicuas hacia el ápice de los lóbulos (Fig. 1 B), la superficie inferior castaña (Fig. 1 C) y la producción de ácido perlatólico como principal sustancia medular.

Canoparmelia scrobicularis (Kremp.) Elix \& Hale difiere por presentar ácido estíctico en la médula ( $\mathrm{K}+$ amarillo), el epitecio frecuentemente pruinoso, y por la forma y longitud de los conidios: en C. austroamericana son bifusiformes a fusiformes y cortos $(5-8 \mu \mathrm{m})$, mientras que en $C$. scrobicularis son filiformes y largos $(18-23 \mu \mathrm{m})$.

2. Canoparmelia caroliniana (Nyl.) Elix \& Hale, Mycotaxon 27: 278. 1986. (Fig. 1 F-I)

Talo folioso, gris mineral, subcoriáceo, corticícola, moderadamente sujeto al sustrato, 3,5-12 cm diám.; lóbulos subirregulares, de ramificación irregular, 1,5-3 $\mathrm{mm}$ de ancho, imbricados, de ápice redondeado; margen entero a crenado, eciliado. Superficie superior lustrosa, lisa a levemente foveolada, con fisuras irregulares a reticulares, maculada; máculas irregulares a reticulares, laminares. Isidios abundantes, laminares, cilíndricos, simples a poco ramificados, a veces coraloides, cortos, con el ápice castaño oscuro a claro, sin cilias, firmes. Soralios, pústulas y dáctilos. Médula blanca. Superficie inferior negra a raramente castaña, lustrosa, lisa a un poco rugosa, continua, a veces con la corteza inferior desprendida, moderada a escasamente ricinada, con una angosta a amplia zona marginal castaña oscura, lustrosa, lisa a rugosa, desnuda a papilada; ricinas simples, negras, a veces grisáceas hacia el extremo, a castañas o blanquecinas, irregularmente distribuidas. Apotecios ausentes. Picnidios ausentes o escasos cuando presentes, submarginales, de ostíolo negro; conidios bifusiformes a baciliformes, 4-6 $\mu \mathrm{m}$ de largo.

Sustancias químicas. Corteza $\mathrm{K}+$ amarillo, UV- [con atranorina (menor)]; médula K-, C-, $\mathrm{KC}+$ rosa, $\mathrm{UV}+$ blanco azulado [con ácidos perlatólico (mayor), estenospórico (menor) y glomeriférico (menor)]. 
Bol. Soc. Argent. Bot. 49 (2) 2014

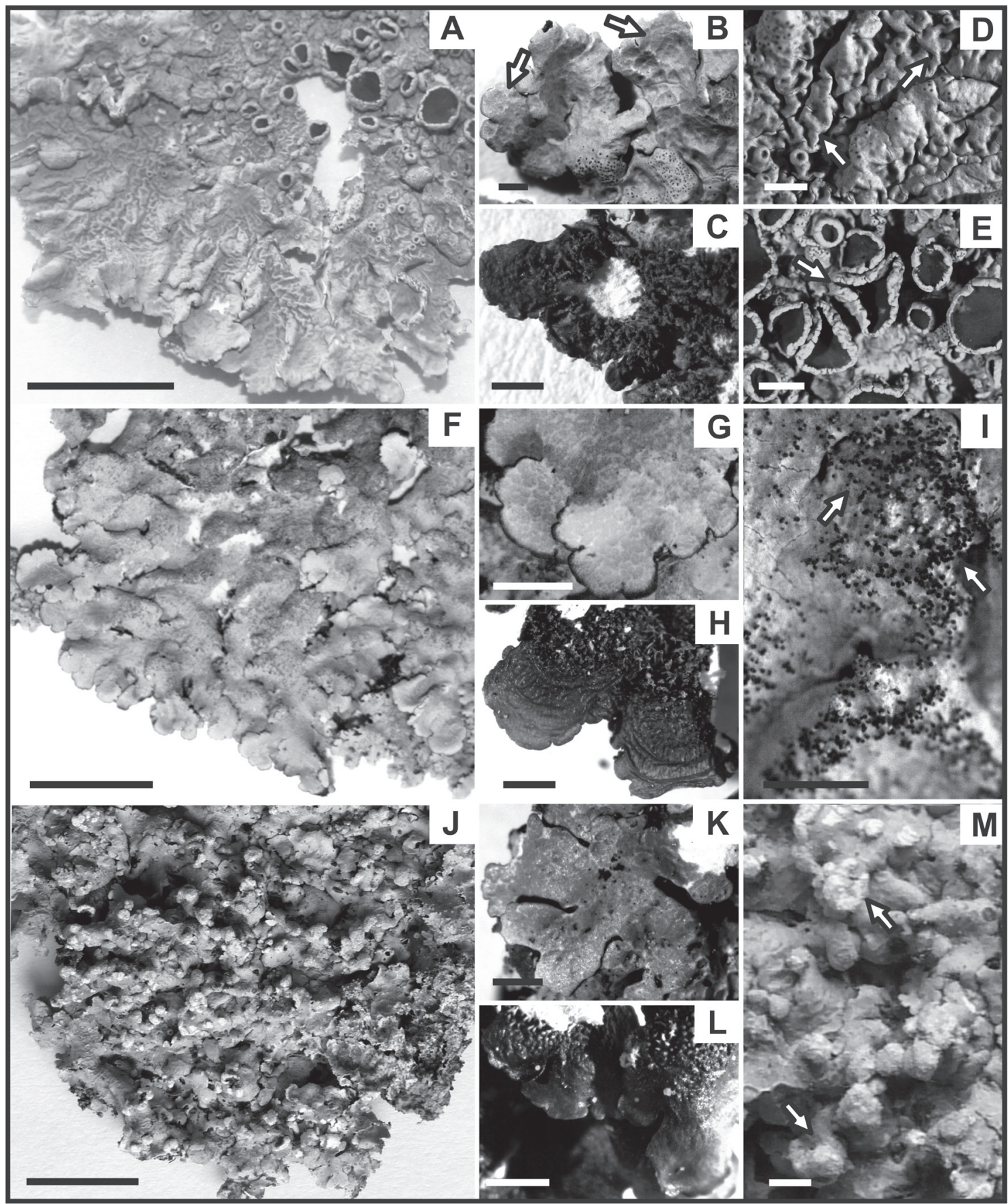

Fig. 1. A-E: Canoparmelia austroamericana. A: Aspecto general del talo. B: Lóbulos en vista superior, mostrando las máculas (flechas). C: Lóbulos en vista inferior. D: Crestas de la superficie superior. E: Apotecios (Michlig \& Niveiro 2309). F-I: Canoparmelia caroliniana. F: Aspecto general del talo (Michlig \& Niveiro 1790). G: Lóbulos en vista superior. H: Lóbulos en vista inferior. I: Isidios con ápice castaño (Michlig et al. 2555). J-M: Canoparmelia cryptochlorophaea. J: Aspecto general del talo. K: Lóbulos en vista superior. L: Lóbulos en vista inferior. M: Soralios capitados (Michlig \& Niveiro 2103). Escalas: A, F, J= 10 $\mathrm{mm}$; B-E, G-I y K-M= $1 \mathrm{~mm}$. 
Material examinado. ARGENTINA. Prov. Misiones: Dpto. Guaraní, Reserva de Biosfera Yaboty, P. P. Caá-Yarí, metros antes de la casa de guardaparques, $26^{\circ} 52^{\prime} 19,3$ " S 54 $14^{\circ}$ ' 32,1" W, $531 \mathrm{msnm}$, sobre Syagrus romanzoffiana, 27/ III/2010, Michlig et al. 2555 (CTES). Dpto. San Pedro, Reserva de Biosfera Yaboty, P. P. Moconá, Embarcadero Piedra Bugre, $27^{\circ}$ 9' $13^{\prime \prime}$ S 53 54' 4" W, 16/V/2008, ipse 913 (CTES); ib., cercanías al ingreso del Sendero Chachi, $27^{\circ} 10,8^{\prime} 53^{\prime \prime} \mathrm{S} 53^{\circ} 54^{\prime}$ 3,1 " W, 326 msnm, sobre ramas caídas al costado del sendero, 24/V/2009, Michlig \& Niveiro 1790 (CTES).

Distribución geográfica. África, América Central (Hale, 1976), del Norte (Hale, 1976; Nash \& Elix, 2002), y Sur, Asia y Europa (Hale, 1976). En América del Sur, se encuentra mencionada para Brasil (Hale, 1976; Spielmann \& Marcelli, 2008), Colombia (Sipman et al., 2008), Ecuador (Hale, 1976) y Venezuela (Hale, 1976; López Figueiras, 1986). Ésta es la primera mención de la especie para la Argentina.

Observaciones. Canoparmelia caroliniana se caracteriza por la superficie superior lisa a levemente foveolada con máculas irregulares a reticulares (Fig. 1 F-G), isidios laminares, abundantes, simples, eciliados con frecuencia con el ápice oscurecido (Fig. 1 I), y ácido perlatólico y glomeriférico en la médula ( $\mathrm{KC}+\mathrm{rosa})$.

Todos los ejemplares estudiados son corticícolas, sin embargo algunos autores mencionan que esta especie puede ser raramente saxícola (Hale, 1976; Nash \& Elix, 2002; Spielmann \& Marcelli, 2008).

El color de la superficie inferior puede variar de castaño claro a oscuro o de castaño oscuro a negro (Eliasaro, 2001), siendo en el material examinado siempre negra en el centro.

En ninguno de los ejemplares estudiados se encontraron apotecios. De acuerdo a Hale (1976) y Nash \& Elix (2002) son raros, laminares, adnatos, de 1-4 mm de diámetro,con un anfitecio usualmente isidiado y ascosporas de 6-8 x 13-15 $\mu \mathrm{m}$.

Canoparmelia caroliniana es la única especie isidiada del género con ácido perlatólico en la médula según expresa Hale (1976) al tratar Pseudoparmelia Lynge. Esta sustancia química se encuentra también en C. aptata (Kremp.) Elix \& Hale, que difiere principalmente por presentar soredios (Hale, 1976).
Otro carácter distintivo de C. caroliniana es la superficie superior fuertemente reticulada, que permite diferenciarla de otras especies isidiadas del género. Canoparmelia amazonica (Nyl.) Elix \& Hale y $C$. concrescens (Vain.) Elix \& Hale carecen de máculas reticulares en la superficie del talo. La primera de ellas difiere además por la producción de ácido protocetrárico (Hale, 1976; Eliasaro, 2001), mientras que la segunda produce ácido divaricático y presenta ascosporas más pequeñas $(5 \times 7-8 \mu \mathrm{m})$ (Hale, 1976).

3. Canoparmelia cryptochlorophaea (Hale) Elix \& Hale, Mycotaxon 27: 278. 1986. (Fig. 1 J-M)

Talo folioso, gris verdoso a veces amarronado, coriáceo a subcoriáceo, corticícola, moderada a débilmente sujeto al sustrato, $4-13 \mathrm{~cm}$ diám.; lóbulos subirregulares, de ramificación irregular, 1-3 mm de ancho, imbricados, de ápice redondeado; margen crenado a crenulado, a veces inciso, eciliado. Superficie superior lustrosa, lisa o rugosa a débilmente foveolada, con fisuras irregulares más frecuentes hacia el centro del talo, con máculas débiles a conspicuas; máculas irregulares a lineares, laminares, más frecuentes hacia la periferia del talo. Soralios presentes, marginales a submarginales, rara vez laminares, capitados; soredios farinosos a granulares, ubicados en los soralios y también frecuentemente dispersos sobre el talo. Isidios, pústulas y dáctilos ausentes. Médula blanca. Superficie inferior negra a castaño oscuro, sublustrosa a lustrosa, lisa a levemente rugosa, moderada a escasamente ricinada, con una angosta zona marginal castaña clara u oscura, rara vez crémea, desnuda a rara vez papilada; ricinas simples, negras, castañas a blanquecinas, distribuidas irregularmente. Apotecios ausentes. Picnidios ausentes o escasos, submarginales, de ostíolo negro; conidios no vistos.

Sustancias químicas. Corteza $\mathrm{K}+$ amarillo, UV- [con atranorina (mayor a menor)]; médula $\mathrm{K}+$ rosa pálido a fuerte o anaranjado pálido tornándose rosa, $\mathrm{C}-\mathrm{o}+$ rosa evanescente, $\mathrm{KC}+$ rosa fuerte persistente o evanescente, a veces tornándose luego anaranjado, UV- [con ácido criptoclorofaeico (mayor) y dos sustancias menores no identificadas de $\mathrm{Rf}=31 \mathrm{y} \mathrm{Rf}=13$ en solvente $\mathrm{C}$ ]. 
Material examinado. ARGENTINA. Prov. Misiones: Dpto. General Manuel Belgrano, alrededores de la Reserva Natural Estricta San Antonio, en predio del INTA, en plantación de Araucaria angustifolia, $26^{\circ} 2$ ' 14,2" S 53 ${ }^{\circ} 47^{\prime}$ 24,8" W, 526 msnm, 29/V/2009, Michlig \& Niveiro 2103 (CTES). Dpto. Iguazú, P. N. Iguazú, Camping Ñandú, 28/IV/2004, Ferraro \& Popoff 7428 (CTES). Dpto. San Ignacio, P. P. Teyú Cuaré, Sendero de la Selva, alrededores del ingreso al camino hacia el Parquizado Inferior, 23/V/2009, Michlig \& Niveiro 1759 (CTES), ib., en un ambiente abierto, poco antes de llegar a la selva, 23/V/2009, ipse 1775 (CTES); ib., mirador Isla Toro, $27^{\circ} 16^{\prime} 55,2$ " S $55^{\circ} 35^{\prime} 40,1$ ' $\mathrm{W}, 155 \mathrm{msnm}, 22 / \mathrm{V} / 2009$, ipse 1696 (CTES). Dpto. San Pedro, Reserva de Biosfera
Yaboty, P. P. Moconá, Embarcadero Piedra Bugre, $27^{\circ}$ 9' 13 " S 53 54' 4" W, 16/V/2008, Michlig et al. 874 (CTES).

Distribución geográfica. América Central (Hale, 1959, 1976), del Norte y Sur, y Asia (Hale, 1976). En América del Sur se encuentra registrada para Brasil (Hale, 1976; Ribeiro, 1998; Jungbluth, 2006) y Venezuela (Hale, 1976). Se registra aquí por primera vez para la Argentina, citando las localidades más australes de la especie.

Observaciones. Canoparmelia cryptochlorophaea es una especie muy característica que presenta soralios capitados marginales a submarginales (Fig. $1 \mathrm{~J}, \mathrm{M}$ ), máculas

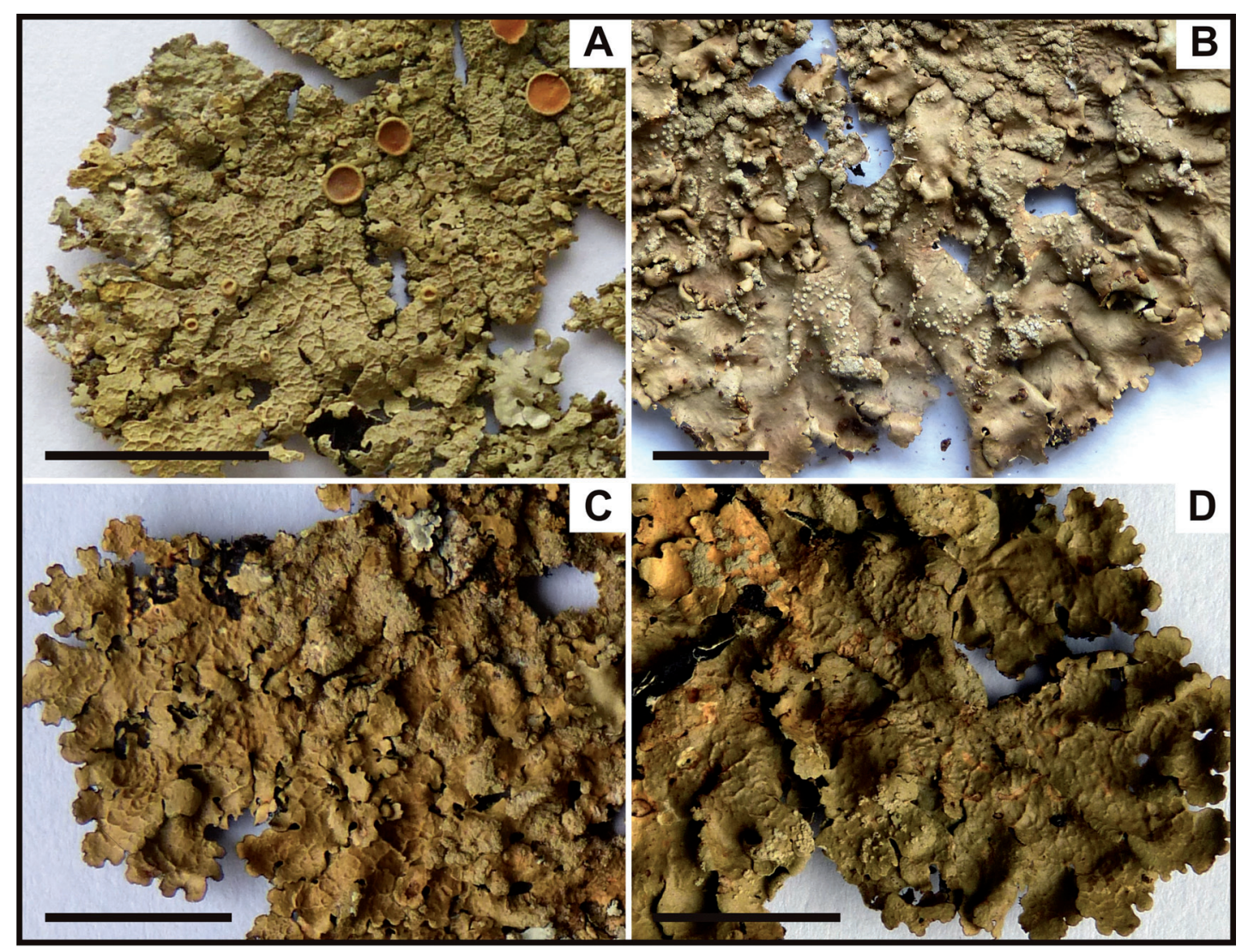

Fig. 2. A: Canoparmelia scrobicularis, aspecto general del talo (Michlig et al. 2722). B: Canoparmelia texana, aspecto general del talo (Michlig et al. 2524). C: Crespoa carneopruinata, aspecto general del talo (Michlig et al. 965). D: Crespoa crozalsiana. aspecto general del talo (Michlig et al. 2520). Escalas= $10 \mathrm{~mm}$. 
irregulares más notorias hacia la periferia de los lóbulos (Fig. $1 \mathrm{~K}$ ) y ácido criptoclorofaeico como principal sustancia medular $(\mathrm{K}+$ rosa pálido, $\mathrm{KC}+$ rosa fuerte). Este ácido es poco frecuente, estando presente además en Cladonia cryptochlorophaea Asahina (Ahti, 2000), Ramalina subfraxinea var. confirmata (Nyl.) G. N. Stevens (Morales Méndez \& Marcano, 1994) y Ramalina aspera Räsänen (Kashiwadani \& Kalb, 1993), asociado a ácido úsnico en las últimas dos especies.

Los apotecios y picnidios normalmente no se encuentran presentes. Hale $(1959,1976)$ fue el único autor que describió la forma y tamaño de las ascosporas, mencionando que éstas son ovoides y miden 4-5 x 6-9 $\mu \mathrm{m}$. Los caracteres de sus conidios nunca fueron descriptos. Ninguno de los picnidios del material estudiado contenía conidios.

4. Canoparmelia scrobicularis (Kremp.) Elix \& Hale, Mycotaxon 27: 279. 1986. (Fig. 2, A)

Talo folioso, gris mineral a gris verdoso, subcoriáceo, corticícola, fuertemente adherido al sustrato, 2-8 cm diám.; lóbulos sublineares, de ramificación irregular a dicotómica anisotómica, 0,5-2 $\mathrm{mm}$ de ancho, contiguos a levemente imbricados, de ápice subtruncado; margen entero a crenado, eciliado. Superficie superior lustrosa, escrobiculada, continua a fisurada a lo largo de las crestas, lisa a levemente rugosa en el centro, maculada; máculas reticulares, distribuidas a lo largo de las crestas. Isidios, soralios, pústulas y dáctilos ausentes. Médula blanca. Superficie inferior negra, lustrosa, lisa a un poco rugosa, continua, moderada a abundantemente ricinada, con una angosta zona marginal castaño claro a oscuro, lustrosa, lisa, desnuda o papilada; ricinas negras, simples a rara vez irregulares, irregular a homogéneamente distribuidas. Apotecios escasos, planos, 0,5-4,5 mm diám., cupuliformes a planos, sésiles, laminares, margen entero a levemente crenado, anfitecio liso, disco castaño claro a oscuro, a veces rojizo, en general con abundante pruina, a veces epruinoso, imperforado; ascosporas ovoides a elipsoidales, 9-15 x 5-10 $\mu \mathrm{m}$. Picnidios presentes, submarginales a laminares, de ostíolo negro; conidios filiformes, a veces curvados, $18-23 \mu \mathrm{m}$ de largo.

Sustancias químicas. Corteza K+ amarillo, UV- [con atranorina (mayor)], médula $\mathrm{K}+$ amarillo, $\mathrm{C}$-, KC-, UV- [con ácidos estíctico (mayor), constíctico (mayor a menor) y menegazziaico (mayor a menor)].

Material examinado. ARGENTINA. Prov. Corrientes: Dpto. Mburucuyá, P. N. Mburucuyá, Sendero Yatay, 20/VII/2006, Michlig et al. 156 (CTES). Prov. Misiones: Dpto. General Manuel Belgrano, Campiña de América, escuela, 26 ${ }^{\circ} 16^{\prime}$ 50,6" S 53 42' 19,4" W, 805 msnm, en selva, al costado del camino, 9/XII/2011, ipse 2722 (CTES). Dpto. Guaraní, Reserva de Biosfera Yaboty, P. P. Caá-Yarí, alrededores de la casa de guardaparques, $26^{\circ} 52^{\prime} 19,6^{\prime \prime} \mathrm{S} 54^{\circ} 13$ ' 33,8" W, $526 \mathrm{msnm}$, sobre tronco de "paraíso" caído, 10/XII/2011, ipse 2782 (CTES). Dpto. San Pedro, Reserva de Biosfera Yaboty, P. P. Esmeralda, camino hacia el arroyo, sobre una rama caída en el interior de la selva, 14/V/2008, ipse 699 (CTES).

Distribución geográfica. Endémica de América del Sur, en donde se encuentra registrada en Brasil, Paraguay (Hale, 1976), Venezuela (Hale, 1976; López Figueiras, 1986) y Argentina, donde fue mencionada para las provincias de Corrientes (Ferraro, 1981; Michlig \& Ferraro, 2012) y Misiones (Osorio, 1969a, 1981; Hale, 1976).

Observaciones. Canoparmelia scrobicularis se caracteriza por la presencia de una superficie superior escrobiculada, apotecios con disco generalmente con abundante pruina, ausencia de propágulos vegetativos y la producción de ácido estíctico como principal sustancia medular ( $\mathrm{K}+$ amarillo).

Canoparmelia austroamericana es la única especie del NEA que comparte con C. scrobicularis la ausencia de propágulos vegetativos. Para información adicional ver las observaciones de $C$. austroamericana.

5. Canoparmelia texana (Tuck.) Elix \& Hale, Mycotaxon 27: 279. 1986. (Fig. 2, B)

Talo folioso, gris mineral, subcoriáceo, corticícola, fuerte a moderadamente sujeto al sustrato, 3-9 mm diám.; lóbulos sublineares a subirregulares, de ramificación irregular a dicotómica anisotómica, 1,5-3 $\mathrm{mm}$ de ancho, imbricados, de ápice redondeado; margen crenado 
a crenulado, con frecuencia oscurecido, eciliado. Superficie superior lustrosa a mate, lisa a rugosa hacia el centro, continua a irregularmente fisurada, emaculada a levemente maculada; máculas débiles, irregulares, hacia la periferia de los lóbulos. Soralios laminares a submarginales, orbiculares a capitados, a veces confluentes; soredios granulares a farinosos. Isidios, pústulas y dáctilos ausentes. Médula blanca. Superficie inferior negra, lustrosa a mate, lisa a poco rugosa, continua, moderadamente ricinada, con una angosta zona marginal castaño oscuro, lustrosa, lisa a rugosa, desnuda a papilada; ricinas simples, negras a amarronadas, a veces con el extremo blanquecino, distribuidas homogéneamente. Apotecios ausentes o rara vez presentes, cuando presentes escasos, 0,7-2 mm diám., adnatos, laminares, margen sorediado, anfitecio liso, disco castaño oscuro, epruinoso, imperforado; ascosporas no vistas. Picnidios ausentes o rara vez presentes, escasos, submarginales a laminares, de ostíolo negro; conidios sublageniformes a débilmente bifusiformes, 5-8 $\mu \mathrm{m}$ de largo.

Sustancias químicas. Corteza K+ amarillo, UV[con atranorina (mayor)]; médula $\mathrm{K}-\mathrm{o}+$ amarillo pálido, $\mathrm{C}+$ rosa pálido o anaranjado pálido, $\mathrm{KC}+$ rosa, UV+ blanco azulado [con ácidos divaricático (mayor) y nordivaricático (menor)].

Material examinado. ARGENTINA. Prov. Chaco: Dpto. Comandante Fernández, Ruta Nac. $N^{\circ}$ 95, entre Presidencia Roque Sáenz Peña y Tres Isletas, kilómetros antes del cruce con la Ruta Prov. N ${ }^{\circ} 44,26^{\circ} 33^{\prime} 44,4$ "S $60^{\circ} 18^{\prime}$ 26,6”W, 108 msnm, en bosquecito de Schinopsis balansae, sobre ramas caídas sobre Cactaceae, 11/II/2010, ipse 2197 (CTES). Dpto. General Güemes, Ruta Prov. $\mathrm{N}^{\circ} 3$, entre Fortín Lavalle y Pampa del

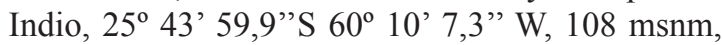
12/II/2010, ipse 2286 (CTES). Dpto. Libertador General San Martín, Ruta provincial No 3, entre Presidencia Roca y la intersección de la Ruta Prov. $\mathrm{N}^{\circ} 90$, camino a la Escuela EGB $\mathrm{N}^{\circ} 564,26^{\circ} 13$, 5"S 59 29' 42,5" W, 75 msnm, 12/II/2010, ipse 2316 (CTES). Dpto. Presidencia de la Plaza, P. N. Chaco, camino a la laguna Panza de Cabra, sobre Prosopis sp., 24/XI/2008, ipse 1103 (CTES). Prov. Corrientes: Dpto. Empedrado, Ruta 12 y arroyo Pehuajó, en isleta de monte degradado por pastoreo, 27/II/2007, Michlig et al. 266 (CTES). Prov. Formosa: Dpto. Bermejo, ruta provincial $\mathrm{N}^{\circ}$ 9, entre Sumayén y El Aibal, $24^{\circ} 22^{\prime} 0,5^{\prime} \mathrm{S}$ $61^{\circ} 38^{\prime} 19,7$ " W, $169 \mathrm{msnm}$, en bosquecito de Aspidosperma quebracho-blanco, 18/IV/2009, ipse 1121 (CTES). Prov. Misiones: Dpto. General Manuel Belgrano, Campiña de América, escuela, $26^{\circ} 16^{\prime} 50,6^{\prime}$ ' S 53 42 ' 19,4" W, 805 msnm, sobre pino al costado del camino, 9/XII/2011, ipse 2713 (CTES). Dpto. Guaraní, Reserva de Biosfera Yaboty, P. P. Caá-Yarí, alrededores de la casa de guardaparques, $26^{\circ} 52^{\prime} 19,6^{\prime \prime} \mathrm{S} 54^{\circ} 13$ ' $33,8^{\prime}$ " W, $526 \mathrm{msm}$, sobre un árbol frente a la casa del guardaparques, 27/III/2010, Michlig et al. 2524 (CTES). Dpto. San Ignacio, P. P. Teyú Cuaré, Sendero de la Selva, alrededores del mirador del río Paraná, sobre Syagrus romanzoffiana, 23/V/2009, Michlig \& Niveiro 1743 (CTES). Dpto. San Pedro, alrededores del P. P. Piñalito, $26^{\circ} 27^{\prime} 28^{\prime \prime} \mathrm{S} 53^{\circ} 52^{\prime}$ 58,7 " W, $768 \mathrm{msnm}$, sobre un poste de alambrado, 9/XII/2011, Michlig et al. 2747 (CTES); Reserva de Biosfera Yaboty, P. P. Moconá, Embarcadero Piedra Bugre, 27 9' 13" S 53 54' 4" W, 16/V/2008, ipse 916 (CTES); $i b$., Sendero Chachi, $27^{\circ} 8,51,5^{\prime \prime} \mathrm{S}$ $53^{\circ} 53^{\prime} 50^{\prime \prime} \mathrm{W}, 341 \mathrm{msnm}$, sobre ramas caídas al costado del sendero, 26/V/2009, Michlig \& Niveiro 1875 (CTES).

Distribución geográfica. África (Hale, 1976), América del Norte (Hale, 1976; Nash \& Elix, 2002), Central (Hale, 1976; Boom et al., 2007) y Sur (Hale, 1976), Asia (Hale, 1976; Kurokawa \& Lai, 2001) y Oceanía (Elix, 1994; Hale, 1976). En América del Sur se encuentra registrada en Argentina (Calvelo \& Liberatore, 2002), Bolivia (Flakus et al., 2011), Brasil (Eliasaro, 2001; Hale, 1976; Jungbluth, 2006; Spielmann \& Marcelli, 2008), Chile (Hale, 1976), Colombia (Sipman et al., 2008), Perú, Uruguay (Hale, 1976) y Venezuela (Hale, 1976; López Figueiras, 1986). En Argentina fue mencionada para las provincias de Buenos Aires (Osorio, 1977; Adler, 1988), Córdoba (Estrabou, 1999), Corrientes (Osorio \& Ferraro, 1975; Ferraro, 1981; Michlig \& Ferraro, 2012), Entre Ríos (Osorio, 1982) y Santiago del Estero (Adler, 2013). Se amplía su distribución a las provincias de Chaco, Formosa y Misiones.

Observaciones. Canoparmelia texana es una especie muy común en el NEA, caracterizada por 
la presencia de soralios laminares, abundantes, orbiculares a capitados, con soredios granulares a farinosos, y ácidos divaricático y nordivaricático en la médula ( $\mathrm{KC}+$ rosa), siendo el rango de variación morfológica que presenta pequeña y su química uniforme y distintiva (Hale, 1976).

La presencia de apotecios y picnidios es rara en esta especie. Solamente en un ejemplar de 18 estudiados fueron encontrados los apotecios pero no contenían ascosporas, mientras que los picnidios fueron encontrados sólo en 3 de ellos. El tamaño de las ascosporas mencionado para la especie es de 8-12 x 5-8 $\mu \mathrm{m}$ (Hale, 1976; Elix, 1994; Eliasaro, 2001; Nash \& Elix, 2002; Jungbluth, 2006).

Canoparmelia texana difiere de otras especies sorediadas de Canoparmelia y Crespoa del NEA por presentar la superficie superior lisa y por la química medular. Crespoa carneopruinata y Cr. crozalsiana, difieren por su superficie superior fuertemente foveolada, y la presencia de ácido estíctico medular y sustancias relacionadas biogenéticamente $(\mathrm{K}+$ amarillo). Canoparmelia cryptochlorophaea se diferencia fácilmente por los soralios notoriamente capitados y ácido criptoclorofaeico en la médula $(\mathrm{K}+$ rosa pálido, $\mathrm{KC}+$ rosa fuerte).

6. Crespoa carneopruinata (Zahlbr.) Lendemer \& Hodkinson, North American Fungi 7(2): 3. 2012. (Fig. 2, C)

Talo folioso, gris mineral a gris verdoso, subcoriáceo, corticícola, fuertemente sujeto al sustrato, 3-8 cm diám.; lóbulos sublineares, de ramificación irregular a dicotómica anisotómica, 0,8-2 mm de ancho, de ápice redondeado, pruinoso; margen crenado, eciliado, oscuro. Superficie superior lustrosa, débil a fuertemente foveolada, con o sin fisuras a lo largo de las crestas, algo rugosa en el centro, maculada; máculas laminares, reticulares en las crestas a irregulares hacia la periferia de los lóbulos. Soralios presentes, laminares, orbiculares, varias veces confluyentes entre sí distribuyéndose a lo largo de las crestas; soredios farinosos a subgranulares. Isidios, pústulas y dáctilos ausentes. Médula blanca. Superficie inferior negra, lustrosa, lisa, moderadamente ricinada, con una angosta zona castaño oscuro en el margen, lustrosa, papilada o desnuda; ricinas simples, negras, distribuidas homogéneamente. Apotecios raramente presentes, escasos, cupuliformes, de
0,8-1 mm diám., laminares, entero, liso, disco imperforado, castaño oscuro a claro, pruinoso; ascosporas no vistas [según Hale (1976) 6-9 x 9-13 $\mu \mathrm{m}]$. Picnidios ausentes.

Sustancias químicas. Corteza K+ amarillo, UV(con atranorina); médula $\mathrm{K}+$ amarillo, C-, KC-, UV[con ácidos estíctico (mayor), constíctico (mayor a menor) y menegazziaico (mayor a menor)].

Material examinado. ARGENTINA. Prov. Corrientes: Dpto. Saladas, Ruta 12, $33.5 \mathrm{~km}$ al $\mathrm{S}$ del desvío a Empedrado, en monte a orillas del camino, alrededores del camping, 27/XII/1983, Ferraro et al. 2848 (CTES). Prov. Misiones: Dpto. Guarani, Reserva de Biosfera Yaboty, P. P. CaáYarí, alrededores de la casa de guardaparques, $26^{\circ} 52^{\prime} 19,6^{\prime}$ S $54^{\circ} 13$ ' 33,8" W, 526 msnm, 27/ III/2010, Michlig et al. 2472 (CTES). Dpto. San Pedro, Reserva de Biosfera Yaboty, P. P. Moconá, alrededores del Centro de Visitantes, $27^{\circ}$ 9' $12,8^{\prime \prime}$ S 53 54' 0,3" W, $314 \mathrm{msnm}$, sobre Syagrus romanzoffiana, 25/V/2009, Michlig \& Niveiro 1821 (CTES); $i b$. cercanías al ingreso del Sendero Chachi, $27^{\circ} 10,8^{\prime} 53^{\prime}$ ' S 53 54' 3, '” W, 326 msnm, 24/V/2009, ipse 1793 (CTES); ib., Sendero La Gruta, $27^{\circ}$ 9' 13 " S 53 54' 4" W, sobre una rama caída, 17/V/2008, Michlig et al. 965 (CTES).

Distribución geográfica. América Central (Hale, 1976; Boom et al., 2007), del Norte (Hale, 1976; Nash \& Elix, 2002) y Sur, Asia, Europa (Hale, 1976). En América del Sur está mencionada para Argentina (Calvelo \& Liberatore, 2002), Brasil (Hale, 1976; Jungbluth, 2006), Colombia (Hale, 1976; Sipman et al., 2008), Uruguay (Hale, 1976) y Venezuela (Hale, 1976; López Figueiras, 1986). En Argentina se encuentra registrada para las provincias de Buenos Aires (Adler, 1988), Córdoba (Estrabou, 1999), Corrientes (Ferraro, 1981; Michlig \& Ferraro, 2012), Entre Ríos (Osorio, 1982), Jujuy (Hale, 1976), Misiones (Osorio, 1981) y Tucumán (Calvelo \& Liberatore, 2002).

Observaciones. Crespoa carneopruinata se caracteriza por la superficie superior foveolada, lóbulos de hasta $2 \mathrm{~mm}$ de ancho, soralios laminares orbiculares a veces confluentes, y por la presencia de ácido estíctico y sustancias relacionadas biogenéticamente en la médula ( $\mathrm{K}+$ amarillo). 
En el material estudiado no se encontraron los picnidios. De acuerdo a Adler (1988) la presencia de estas estructuras son raras en la especie, los conidios son filiformes y de 11-16 $\mu \mathrm{m}$ de largo. En cuanto a los apotecios, sólo uno fue observado y no contenía ascosporas maduras.

Una especie muy afín a ésta es Cr. crozalsiana, también presente en el NEA, que difiere únicamente en el ancho de los lóbulos (2-4 mm). Existen algunas controversias en cuanto a la delimitación de ambas: mientras algunos autores consideran que deberían tratarse como sinónimos (Estrabou, 1999; McCarthy, 2012), otros consideran que ambas son especies diferentes (Hale, 1976; Junbgluth, 2006; Spielmann \& Marcelli, 2008; Hawksworth, 2011; Lendemer \& Hodkinson, 2012). En este trabajo, se consideran ambas especies como diferentes, ya que el material estudiado permitió una clara delimitación entre ambas. Crespoa carneopruinata es más común en áreas de mayor humedad, mientras que Cr. crozalsiana es más común en ambientes más secos.

7. Crespoa crozalsiana (B. de Lesd.) Lendemer \& Hodkinson, North American Fungi 7 (2): 3. 2012. (Fig. 2, D)

Talo folioso, gris mineral a gris verdoso, coriáceo, corticícola, fuertemente sujeto al sustrato, 7-12 cm diám.; lóbulos subirregulares, de ramificación irregular, 2-4 $\mathrm{mm}$ de ancho, contiguos a levemente imbricados, de ápice redondeado; margen irregularmente inciso, eciliado, castaño. Superficie superior lustrosa, foveolada, con crestas formando retículos, con fisuras en las crestas, maculada; máculas laminares, reticulares en las crestas e irregulares hacia la periferia de los lóbulos. Soralios laminares, abundantes, circulares, frecuentemente producidos sobre las crestas, muchas veces coalescentes; soredios granulares. Isidios, pústulas y dáctilos ausentes. Médula blanca. Superficie inferior negra, lustrosa, lisa, abundantemente ricinada, con una angosta zona marrón oscura desnuda hacia el margen, lustrosa, lisa; ricinas generalmente simples, a veces irregulares, negras, a veces con el extremo castaño, de distribución homogénea. Apotecios generalmente ausentes, cuando presentes escasos, cupuliformes a planos, de 3,5 mm diám., sésiles, laminares, margen entero a levemente crenado, anfitecio liso, disco imperforado, castaño oscuro, pruinoso; ascosporas elipsoidales, $8-13$ x (4) 6-8 $\mu \mathrm{m}$. Picnidios ausentes.

Sustancias químicas. Corteza K+ amarillo, UV(con atranorina); médula $\mathrm{K}+$ amarillo, C-, KC-, UV[con ácidos estíctico (mayor), constíctico (mayor a menor) y menegazziaico (mayor a menor)].

Material examinado. ARGENTINA. Prov. Corrientes: Dpto. Mburucuyá, P. N. Mburucuyá, Sendero Che-Roga, en isleta de bosque higrófilo, 6/IV/2007, Michlig \& Niveiro 347 (CTES). Prov. Formosa: Dpto. Pirané, ruta provincial $\mathrm{N}^{\circ} 1$, entre Mayor Villafañe y San Francisco de Laishi, $26^{\circ} 12$ ' 16,8" S $58^{\circ} 59^{\prime} 47,3$ " W, 73 msnm, 12/ II/2010, ipse 2338 (CTES). Prov. Misiones: Dpto. Guaraní, Reserva de Biosfera Yaboty, P. P. CaáYarí, alrededores de la casa de guardaparques, $26^{\circ}$ 52 ' 19,6 " S $54^{\circ} 13^{\prime} 33,8^{\prime}$ ' W, $526 \mathrm{msnm}$, sobre un árbol frente a la casa del guardaparques, 27/ III/2010, Michlig et al. 2520 (CTES), ib., sobre duraznero, 10/XII/2011, ipse 2801 (CTES). Dpto. San Pedro, Reserva de Biosfera Yaboty, P. P. Moconá, alrededores del Centro de Visitantes, $27^{\circ} 9^{\prime} 12,8^{\prime}$ ' S 53 54' 0,3" W, 314 msnm, sobre Syagrus romanzoffiana, 25/V/2009, Michlig \& Niveiro 1824 (CTES).

Distribución geográfica. África (Hale, 1976), América del Norte (Hale, 1976, Nash \& Elix, 2002) y Sur, Europa (Hale, 1976) y Oceanía (Elix, 1994). En América del Sur se encuentra registrada en Argentina (Hale, 1976), Brasil (Hale, 1976; Spielmann \& Marcelli, 2008), Uruguay (Hale, 1976) y Venezuela (López Figueiras, 1986). En Argentina se encuentra mencionada para las provincias de Buenos Aires (Osorio, 1977; Adler, 1988), Corrientes (Ferraro, 1981; Michlig \& Ferraro, 2012), Misiones (Osorio, 1969b), Santiago del Estero (Adler, 2013) y Tucumán (Hale, 1976). Se extiende su distribución a la provincia de Formosa.

Observaciones. Crespoa crozalsiana se caracteriza por la superficie superior foveolada, lóbulos de 2-4 mm de ancho, soralios laminares orbiculares a veces confluentes, y por la presencia de ácido estíctico y sustancias asociadas en la médula ( $\mathrm{K}+$ amarillo). 
Esta especie está estrechamente relacionada a Cr. carneopruinata, de la cual difiere únicamente por presentar lóbulos más anchos (2-4 mm). Para información adicional ver las observaciones de $\mathrm{Cr}$. carneopruinata.

\section{Agradecimientos}

A la Administración de Parques Nacionales (APN) y al Ministerio de Ecología, Recursos Naturales Renovables y Turismo (MERNRyT) de la provincia de Misiones por los permisos de colección y a los guardaparques por la asistencia brindada durante los viajes de campaña. Este trabajo es parte de la tesis doctoral de la autora, presentada en la Facultad de Ciencias Exactas, Físicas y Naturales de la Universidad Nacional de Córdoba. Fue financiado por el Consejo Nacional de Investigaciones Científicas y Técnicas (CONICET), la Secretaría General de Ciencia y Técnica (SGCyT-UNNE), Myndel Botanica Foundation y MERNRyT (Proyecto Araucaria XXI).

\section{Bibliografía}

ADLER, M. T. 1987. A new species of the genus Canoparmelia from Argentina. Mycotaxon 28: 251254.

ADLER, M. T. 1988. La familia Parmeliaceae (líquenes, Ascomycotina) en la provincia de Buenos Aires: estudio taxonómico-florístico. Tesis Doctoral. Universidad de Buenos Aires. 236 págs.

ADLER, M. T. 2013. Líquenes Parmelioides (Parmeliaceae, Ascomycota) del Parque Nacional Copo (Provincia de Santiago del Estero, Argentina). Bol. Soc. Argent. Bot. 48: 387-406.

AHTI, T. 2000. Cladoniaceae. Flora Neotropica Monogr. 78: $1-362$.

BOOM, P. P., G. VAN DEN, J. A. ELIX \& H. J. M. SIPMAN. 2007. New or interesting lichen records from Guatemala I. Wildenowia 37: 365-375.

CALVELO, S. \& S. LIBERATORE. 2002. Catálogo de los líquenes de la Argentina. Kurtziana 29: 7-170.

CRESPO, A., F. KAUFF, P. K. DIVAKAR, R. DEL PRADO, S. PÉREZ-ORTEGA, G. AMO DE PAZ, Z. FERENCOVA, O. BLANCO, B. ROCAVALIENTE, J. NÚÑEZ-ZAPATA, P. CUBAS, A. ARGÜELLO, J. A. ELIX, T. L. ESSLINGER, D. L. HAWKSWORTH, A. MILLANES, M. C. MOLINA,
M. WEDIN, T. AHTI, A. APTROOT, E. BARRENO, F. BUNGARTZ, S. CALVELO, M. CANDAN, M. COLE, D. ERTZ, B. GOFFINET, L. LINDBLOM, R. LÜCKING, F. LUTZONI, J. E. MATTSSON, M. I. MESSUTI, J. MIADLIKOWSKA, M. PIERCEYNORMORE, V. J. RICO, H. J. M. SIPMAN, I. SCHMITT, T. SPRIBILLE, A. THELL, G. THOR, D. K. UPRETI \& H. T. LUMBSCH. 2010a. Phylogenetic generic classification of parmelioid lichens (Parmeliaceae, Ascomycota) based on molecular, morphological and chemical evidence. Taxon 59: 1735-1753.

CRESPO, A., Z. FERENCOVA, S. PÉREZORTEGA, J. A. ELIX \& P. K. DIVAKAR. 2010b. Austroparmelina, a new Australasian lineage in parmelioid lichens (Parmeliaceae, Ascomycota). Syst. Biodivers. 8: 209-221.

CRESPO, A., P. K. DIVAKAR \& D. L. HAWKSWORTH. 2011. Generic concepts in parmelioid lichens, and the phylogenetic value of characters used in their circumscription. Lichenologist 43: 511-535.

ELIASARO, S. 2001. Estudio taxonómico y florístico sobre las Parmeliaceae sensu stricto (Ascomycota liquenizados) del Segundo Planalto del Estado de Paraná, Brasil. Tesis doctoral. Universidad de Buenos Aires. 281 págs.

ELIX, J. A. 1993. Progress in the generic delimitation of Parmelia sensu lato lichens (Ascomycotina: Parmeliaceae) and a synoptic key to the Parmeliaceae. Bryologist 96: 359-383.

ELIX, J. A. 1994. Canoparmelia. In Flora of Australia, Lichens. Introduction, Lecanorales 55: 21-31. Australia Government Publishing Service, Canberra.

ELIX, J. A., J. JOHNSTON \& D. VERDON. 1986. Canoparmelia, Paraparmelia and Relicinopsis, three new genera in the Parmeliaceae (lichenized Ascomycotina). Mycotaxon 27: 271-282.

ESTRABOU, C. 1999. La familia Parmeliaceae (Ascomycetes liquenizados) sensu stricto de la provincia de Córdoba: estudio sistemáticobiogeográfico. Tesis Doctoral. Facultad de Ciencias Exactas, Físicas y Naturales, Universidad Nacional de Córdoba. 158 págs.

FERRARO, L. I. 1981. Contribución al estudio de las Parmeliáceas (líquenes) de Corrientes, Rep. Argentina. Bonplandia 5: 83-99.

FLAKUS, A., M. OSET, A. JABLOŃSKA, P. RODRIGUEZ SAAVEDRA \& M. KUKWA. 2011. Contribution to the knowledge of the lichen biota of Bolivia, 3. Polish Bot. J. 56: 159-183.

HALE, M. E. 1959. New or interesting species of Parmelia in North America. Bryologist 62: 16-24.

HALE, M. E. 1974. New combinations in the lichen genus Pseudoparmelia Lynge. Phytologia 29: 188191. 
HALE, M. E. 1976. A monograph of the lichen genus Pseudoparmelia Lynge (Parmeliaceae). Smithsonian Contr. Bot. 31: 1-62.

HAWKSWORTH, D. L. 2011. Parmotrema subgen. Crespoa subgen. nov. for the Canoparmelia crozalsiana clade. Lichenologist 43: 647-648.

JUNGBLUTH, P. 2006. A família Parmeliaceae (fungos liquenizados) em cerrados do Estado de São Paulo, Brasil. Dissertação (mestrado). Instituto de Botânica da Secretaria de Estado do Meio Ambiente. São Paulo, Brasil. 323 págs.

KASHIWADANI, H. \& K. KALB. 1993. The genus Ramalina in Brazil. Lichenologist 25: 1-31.

KUROKAWA, S. \& M. J. LAI. 2001. Parmelioid lichens genera and species in Taiwan. Mycotaxon 77: 225 284.

LENDEMER, J. C. \& B. P. HODKINSON. 2012. Recognition of the Parmelia crozalsiana group as the genus Crespoa. North American Fungi 7: 1-5.

LÓPEZ FIGUEIRAS, M. 1986. Censo de macrolíquenes venezolanos de los estados Falcón, Lara, Mérica, Tachira y Trujillo. Universidad de los Andes, Facultad de Farmacia. 521 pp.

McCARTHY, P. M. 2012. Checklist of the Lichens of Australia and its Island Territories [online]. Australian Biological Resources Study, Canberra. Disponible en:http://www.anbg.gov.au/abrs/ lichenlist/introduction.html [Acceso: 16 Marzo 2005].

MICHLIG, A. \& L. FERRARO. 2012. Diversidad de macrolíquenes del Parque Nacional Mburucuyá (Corrientes, Argentina). Bol. Soc. Argent. Bot. 47: 287-302.

MORALES MÉNDEZ, A. \& V. MARCANO. 1994. Ramalina subfraxinea Nyl. var. confirmata (Nyl.) N. Stevens (Ascomicete liquenizado, Ramalinaceae) en América. Trop. Bryol. 9: 201-204.

NASH I. I. , T. H. \& J. A. ELIX. 2002. Canoparmelia. En: Nash III, T. H., B. D. Ryan, C. Gries \& F. Bungartz (eds.), LICHEN FLORA OF THE GREATER SONORAN DESERT REGION, Arizona State University, Vol. 1: 122-125. Lichens Unlimited, Arizona State University, Arizona.

ORANGE, A., P. W. JAMES \& F. J. WHITE. 2010. Microchemical methods for the identification of lichens. $2^{\text {nd }}$ Edition, British Lichen Society. $101 \mathrm{pp}$.

OSORIO, H. S. 1969a. Contributions to the lichen flora of Argentina, II. Lichens from the province of Misiones. Comun. Bot. Mus. Hist. Nat. Montevideo 4: $1-5$.

OSORIO, H. S. 1969b. Contribution to the lichen flora of Argentina. III. Additions. Bryologist 72: 409-410.
OSORIO, H. S. 1977. Contributions to the lichen flora of Argentina X. Lichens from Santa Catalina, Buenos Aires Province. Bryologist 80: 363-364.

OSORIO, H. S. 1981. Contribution to the lichen flora of Argentina XIII. Lichens from Misiones province. Comun. Bot. Mus. Hist. Nat. Montevideo 4(63): $1-18$.

OSORIO, H. S. 1982. Contribution to the lichen flora of Argentina XIV. Lichens from Entre Ríos province. Comun. Bot. Mus. Hist. Nat. Montevideo 4: 1-8.

OSORIO, H. S. \& L. I. FERRARO. 1975. Contributions to the lichen flora of Argentina, VII. New and noteworthy records from the province of Corrientes. Comun. Bot. Mus. Hist. Nat. Montevideo 4(57): 1-4.

QUILHOT, W., I. PEREIRA, G. GUZMÁN, R. RODRÍGUEZ \& T. SEREY. 1998. Categorías de conservación de líquenes nativos de Chile. Bol. Mus. Nac. Hist. Nat., Santiago de Chile 47: 9-22.

RIBEIRO, C. H. 1998. A família Parmeliaceae (Ascomycota liquenizados) em regiões montanhosas dos estados de Minas Gerais, Rio de Janeiro e São Paulo. Dissertação (mestrado). Instituto de Biociências da Universidade de São Paulo. 194 págs.

SIPMAN, H. J. M., W. HEKKING \& C. J. AGUIRRE. 2008. Checklist of lichenized and lichenicolous fungi from Colombia. Bibl. J. J. Triana 20: 1-242.

SPIELMANN, A. A. \& M. P. MARCELLI. 2008. Parmeliaceae (Ascomycota liquenizados) nos barrancos e peraus da encosta da Serra Geral, Vale do Rio Pardo, Rio Grande do Sul, Brasil. II. Gêneros Canoparmelia, Hypotrachyna, Myelochroa, Parmelinopsis e Relicina. Iheringia, Bot. 63(2): 193212.

THE INTERNATIONAL PLANT NAMES INDEX (2012). Disponible en: http://www.ipni.org [Acceso: Julio 2013].

THIERS, B. 2013. Index Herbariorum: A global directory of publica herbaria and associated staff [online]. Disponible en: http://sweetgum.nybg.org/ih/ [Acceso: Julio 2013].

WHITE, F. J. \& P.W. JAMES.1985. A new guide to microchemical tecniques for the identification of the lichen substances. Bull. Brit. Lichen. Soc. 57 (suppl.): 1-41.

Recibido el 12 de agosto de 2013, aceptado el 22 de abril de 2014. 